\title{
Influence of nucleon and pion mean fields of the description of spallation reactions
}

\author{
Th. Aoust ${ }^{1,2}$, A. Boudard ${ }^{3}$, J. Cugnon ${ }^{2, a}$, S. Leray ${ }^{3}$, and Y. Yariv ${ }^{3,4}$ \\ 1 SCK-CEN, Boeretang 200, $2400 \mathrm{Mol}$, Belgium \\ 2 Physics Department, University of Liège, Allée du 6 Août 17, Bât. B5, 4000 Liège 1, Belgium \\ 3 DAPNIA/SPhN, CEA Saclay, 91191 Gif-sur-Yvette Cedex, France \\ 4 Soreq NRC, Yavne 81800, Israel
}

\begin{abstract}
Intranuclear cascade plus evaporation models are able to describe spallation reactions using very few input, among which a mean field for nucleons and for pions, generally under the form of crude potential wells. The influence of this ingredient, especially of the isospin and the energy-dependence of the nucleon mean field is investigated. The influence of the presence of a pion mean field is also studied.
\end{abstract}

\section{Introduction}

Spallation reactions induced by nucleons and light nuclei are best described by intranuclear cascade (INC) plus evaporation models. Their predictions are more and more accurate (in the $200 \mathrm{MeV}-2 \mathrm{GeV}$ range) in spite of the fact that INC models are rather crude: they indeed reduce the collision process to a quasi-classical multiple scattering occurring in a Fermi gas of nucleons contained in a potential well. In the last two decades, the INC models have been refined continuously, but their ingredients still remain very simple: nucleon-nucleon cross sections plus a handful set of parameters defining minimally the target structure. In this paper we pay attention to the average nucleon and pion mean fields. Usually, the nucleon mean field is described by a constant static potential well. In some cases, a different depth of the potential well is introduced for protons and neutrons. However, the accumulated phenomenology of the optical-model potential indicates that the depth should be energy-dependent [1], a feature which is completely neglected by INC models. Furthermore, the pion mean field is always treated very crudely, if not neglected. We want here to discuss the influence of introducing more realistic mean fields, keeping in mind that simulation models like INC cannot handle easily complicated features. Our methodology is the following. We will start which the INCL4.2 version [2] of the Liège INC model, which has the same potential for neutrons and protons and no pion potential, and add successively the isospin-dependence and energy-dependence of the nucleon mean field, and an isospin-dependent mean field for the pions. We will discuss the effects of these modifications on the main experimental observables.

\section{Phenomenology of the nucleon and pion mean fields}

\subsection{Nucleons}

The nucleon mean field is usually identified as the real part of the optical-model potential for positive energy and to the

\footnotetext{
a Presenting author, e-mail: J.Cugnon@ulg.ac.be
}

shell-model potential for negative energy [3]. There is a continuity between these two quantities, which can be unified by the concept of the mass operator for quasi-particle excitations, introducing a mathematical link between them under the form of a dispersion relation, which naturally introduces the energydependence. The phenomenological energy-dependence is basically contained in the following form for the depth $(i=$ $p, n)$ :

$$
V(E)=V_{0}^{i}+\alpha_{i}\left(E-E_{F}^{i}\right) .
$$

The potential and total energies are also related by

$$
E=\frac{\hbar^{2} k^{2}}{2 m}+V(E)
$$

which involves a modification of the energy-momentum relation. The quantities $E_{F}^{i}$ are the Fermi energies. The potential depths at the Fermi energy, $V_{0}^{i}$, are related to the separation energies by $-S_{i}=T_{F}^{i}+V_{0}^{i}$, where $T_{F}$ is the Fermi kinetic energy. The quantities $\alpha_{i}$ are close to $-0.23 \mathrm{MeV}^{-1}$. Therefore the potentials given in equation (1) vanish for an energy $\mathrm{E} \sim 200 \mathrm{MeV}$. Above these value, the mean field basically vanishes. We will also consider energy-independent potentials (by taking $\alpha_{i}=0$, but keeping different $V_{0}^{i}$,s), that we will refer to as isospin-dependent potentials.

\subsection{Pions}

The phenomenology of the pion mean field is a more complicated question. What is of interest for INC models is the potential energy of a pion inside matter. One may try to identify this quantity with the real part of the pion optical-model potential, as for the nucleon case. However, the imaginary part of the pion optical-model potential is very large in the so-called $\Delta$-resonance region, which is relevant for spallation reactions. This means that pions are strongly absorbed by nuclei. More exactly, it means that the incoming flux in the elastic channel will not penetrate the nucleus. As a consequence, the real part of the pion optical-model potential is badly determined in the nuclear interior, as it is well known. However, in nucleoninduced spallation reactions, a pion can be created inside the 
nucleus and the question of its potential energy inside the nucleus is raised. Obviously, this question cannot be answered by relying on the pion optical-model, since the latter describes the evolution of the incoming flux in the elastic channel. The reason is that, contrarily to the nucleons, pions are not good quasi-particle excitations of nuclei. In the former case, the imaginary part of the nucleon optical-model potential is rather small, nucleons can propagate freely (for a few fm's) inside matter and thus have a rather well-defined potential energy. This property is embodied by the so-called strength function $S(k, \omega)$, which grossly speaking is the probability for an excitation of momentum $k$ to have an energy $\omega$. It is roughly related to the optical-model potential $V+i W$ by the relation:

$$
S(k, \omega)=-\frac{1}{\pi} \frac{W}{\left[\omega-\hbar^{2} k^{2} / 2 m-V\right]^{2}+[W]^{2}} .
$$

If $W$ is very small, the function $S$ differs from zero only if $\omega \approx \hbar^{2} k^{2} / 2 m+V$, meaning that the quasi-particle energy is rather well defined and its potential energy is given by the real part of optical-model potential. On the other hand, if $W$ is very large, as in the pion case, $S$ is different from zero in a very broad range. This is of course consistent with the Heisenberg principle: the quasi-particle has a lifetime of the order of $\hbar / W$ and, therefore its energy has a fluctuation of the order of $W$. For pions, $W$ may be as large as $200 \mathrm{MeV}$, and the definition of the their potential energy may have no real sense. Note that this effect, though smaller, is not of negligible size for nucleons. See ref. [4] for an interesting discussion. The very success of INC models indicates that treating these nucleons on shell, i.e. with their average potential energy is probably a good approximation. It is hoped that the same approximation could be adopted for pions.

The choice of the potential energy remains however. One can be helped by the fact the real part of the pion optical-model potential is nevertheless well determined in the nuclear surface region, as it is discussed in ref. [5]. Here, we will follow the same pragmatic approach as in this reference, using a constant square well potential for the nuclear part of the pion mean field extending up to a radial distance $r_{\text {max }}=R_{0}+c$, with a depth $\mathrm{V}$ exhibiting the following Lane form:

$$
V=V_{1}+V_{2} \tau_{3}(N-Z) / A,
$$

where $\tau_{3}$ is the third isospin component of the pion, and where $\mathrm{N}, \mathrm{Z}$ and $\mathrm{A}$ refer to the target. The parameters $V_{1}, V_{2}$ and $c$ are determined by a fit of the INC calculations on some key measurements, involving total pion cross sections in protoninduced reactions and pion absorption cross sections. Their numerical values are $V_{1}=-31 \mathrm{MeV}, V_{2}=-71 \mathrm{MeV}$ and $c=1.8 \mathrm{fm}$, respectively. These values are consistent with the phenomenological values of the pion optical-model potential in the surface region.

\section{Influence of the description of the mean field}

We here present calculations of spallation reactions using the Liège INC model, with different choices for the mean field. We refer to the INCL4.2 model, described in detail in ref. [2], as the standard case. We add successively an isospin dependent potential, an isospin and energy-dependent potential for nucleons, and finally the potential described above for the pions.

\subsection{Particle multiplicities}

The effect of the choice of the potential for the nucleons on particle multiplicities is illustrated by table 1 . Using an isospin-dependent potential diminishes the cascade neutron multiplicity and increases the proton multiplicity (dominated by cascade). This is due to the fact that, for an asymmetric nucleus, the neutron well depth is increased and the proton well depth is decreased. The neutron evaporation rate is slightly increased, because the excitation energy is slightly increased as a mere consequence of the global decrease of the nucleon emission in the cascade stage. Adding the energydependence of the nucleon mean field decreases both neutron and proton emission in the cascade stage (and increases the excitation energy and the evaporation of neutrons) because nucleons are then more bound, on the average. The introduction of the pion potential only slightly affects nucleon emission in the cascade stage, but still increases the excitation energy, since low-energy pions cannot escape freely and are trapped in the target and finally absorbed. It is interesting to note that the introduction of the new average potentials drives the prediction for the cascade neutron multiplicity close the experimental value. Unfortunately, it somehow destroys the good agreement for the evaporated nucleons, although this quantity may be sensitive to the detail of the evaporation code.

\subsection{Nucleon double differential cross sections}

The shape of the neutron and proton double differential cross sections remain globally unchanged by the modifications described above. Nevertheless, systematic limited changes are worth to be mentioned. First, the quasi-elatic peak in neutron spectra is better reproduced than with the standard version: it is broadened and its maximum is shifted towards lower energy and comes closer to the experimental data. Second, the quasi-elastic peak in proton spectra is broadened. Third, the neutron spectra are slightly depleted in the $20-50 \mathrm{MeV}$. This change is sometimes an improvement and sometimes not (details can be found in ref. [7], where a partial account of this work is provided). These trends are mainly due to the the

Table 1. Particle multiplicities and excitation energy $E^{\star}$ in $\mathrm{p}^{208} \mathrm{~Pb}$ collisions at $800 \mathrm{MeV}$. Comparison between the predictions of the standard INCL4 model, of the versions including successively the isospin and energy-dependencies for the nucleons and the potential for pions, and the experimental data from ref. [6].

\begin{tabular}{cccccc}
\hline & experiment & INCL4 & $+\mathrm{V}(\tau)$ & $+\mathrm{V}(\tau, \mathrm{E})$ & $\mathrm{V}_{\pi}$ \\
\hline $\mathrm{n} 2-20 \mathrm{MeV}$ & $6.5 \pm 0.7$ & 6.81 & 6.97 & 7.01 & 7.43 \\
$\mathrm{n}>20 \mathrm{MeV}$ & $1.9 \pm 0.2$ & 2.49 & 2.28 & 2.22 & 2.22 \\
$\mathrm{p}$ & & 2.56 & 2.70 & 2.65 & 2.72 \\
$\mathrm{E}^{\star}(\mathrm{MeV})$ & & 119.9 & 123.1 & 126.9 & 136.3 \\
\hline
\end{tabular}


introduction of the energy-dependence of the nucleon mean field. This changes modifies the blocking of the quasi-elastic $(\mathrm{p}, \mathrm{n})$ collisions (and the quasi-elastic peak) and enhances the average binding energy of the neutrons, making more difficult the ejection of low-energy neutrons.

\subsection{Residue cross sections}

The changes on the residue production cross sections are also limited. There is a slight broadening of the spallation peak of the residue mass spectrum, but not enough to cure the lack of cross section in the low mass range of this peak in the standard version [2]. This change is of course related to the slight increase of the excitation function (see table 1). In addition, there seems to be a slight decrease in the production of the residues with small mass loss.

Let us however mention that the production of residues with one more nucleon (like ${ }^{210} \mathrm{Po}$ in $\mathrm{p}-{ }^{209} \mathrm{Bi}$ interactions) can be drastically changed. Since they can be formed by $(p, \pi)$ reactions, they are sensitive to the pion potential. See ref. [8] for a discussion of this particular case.

\subsection{Pion-induced reactions}

Since the most drastic modifications introduced in the INCL4 model involves pions (their mean field was neglected in the standard version), it is interesting to investigate these reactions first.

The total reaction cross sections are not very much changed by the modifications introduced in this work. This is rather expected, since what matters for this observable is the first collision and since the pion-nucleon cross section has not been modified in the $\Delta$-resonance region and below. Only the modification of the nucleon potential slightly modifies the kinematics of this first collision. We observed a slight improvement, as reported on in ref. [5]. But, both with the standard and the modified versions, the reaction cross section is underpredicted below $\Delta$-resonance, at least for heavy targets. This can be attributable to the interaction of low-energy pions with two nucleons, which is neglected in INCL4.

A more sensitive test is provided by (true) absorption cross sections. A sample of our results is given in figure 1. Clearly, in this case, the predictions of INCL4 are improved. The main source of the improvement comes from the introduction of the pion mean field. It is then harder to emit a low-energy pion, which has thus more chance to be annihilated on nucleons. This is on keeping with the smaller improvement that we observed for negative pions. Indeed the latter experience a somehow deeper potential well inside the nucleus, but no Coulomb barrier when they leave this nucleus. We also obtained less satisfactory results for very heavy nuclei. This could be understood as due to the large probability for direct pion annihilation on two nucleons, due to the large size of its de Broglie wavelength, an aspect completely forgotten in INCL4. Nevertheless the good overall results and the presence of the $\Delta$-peak in the absorption cross sections indicates that our modelisation of the pion-nucleus intera ction has probably

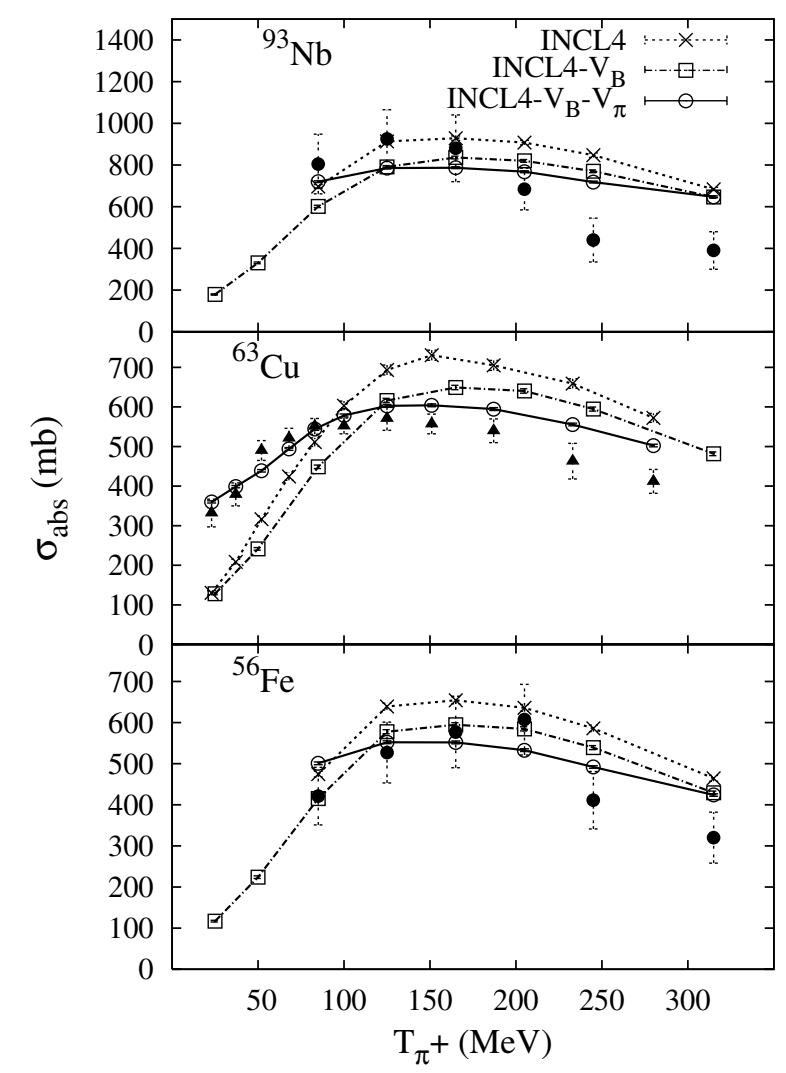

Fig. 1. True absorption cross section of positive pions on indicated targets, as function of the pion incident energy. Comparison between the experimental data of refs. $[9,10]$ (heavy symbols), the predictions of the standard INCL4 model (dotted lines with crosses) and those of the modified models, including isospin and energy-dependance of the nucleon average potential (dot-dashed lines with open squares) and also the pion mean field (full lines with open circles).

picked up the right features. This is corroborated by the satisfactory results that we obtained (slightly better in the standard version) for proton production in pion-induced reactions (see ref. [5] for a preliminary account of this point).

\subsection{Pion production in proton-induced reactions}

Let us look first to the integrated pion production first. Typical results of our study are given in figure 2 . The cross sections are markedly overestimated by the standard version of INCL4, the well-known shortcoming of this model [2]. When the isospin and especially energy-dependence of the nucleon mean field are introduced, this defect is largely alleviated (almost totally for light targets). We think that this may be due to an effective modification of the threshold for pion production. Indeed the continuous rising of the pion production cross section mainly results from the rising elementary $\mathrm{NN}$ cross section in the energy range under consideration. When an energy-dependent potential is used, the incoming proton has inside the nucleus a kinetic energy equal to its incident energy (above $200 \mathrm{MeV}$ ). When a constant potential is used, this kinetic energy is increased by the depth of the potential, say $50 \mathrm{MeV}$. This could explain the roughly constant shift between the dotted and the 


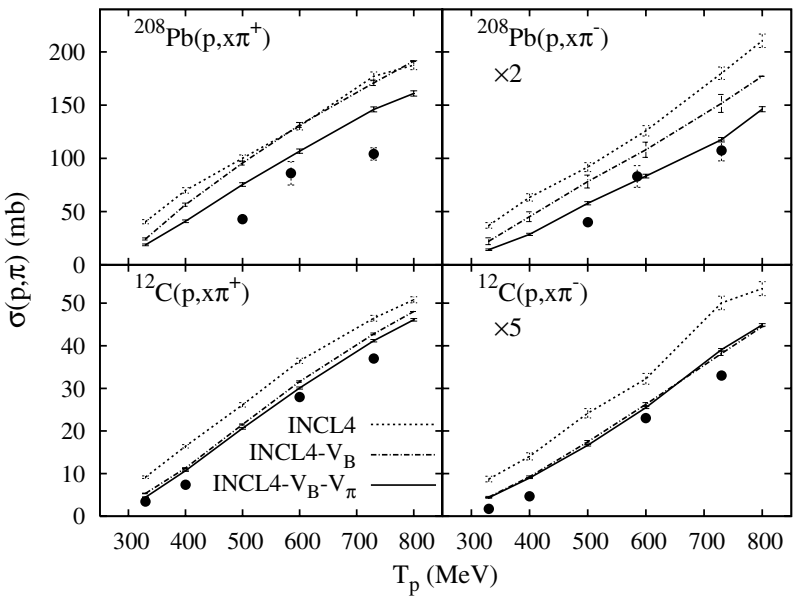

Fig. 2. Positive (left) and negative (right) pion production cross sections in proton-induced collisions on ${ }^{208} \mathrm{~Pb}$ (upper panels) and ${ }^{12} \mathrm{C}$ (lower panels) nuclei, as functions of the incident proton kinetic energy $T_{p}$. Comparison between the experimental data of refs. $[9,10]$ (heavy dots), the predictions of the standard INCL4 model (dotted lines) and those of the modified model, including isospin and energydependance of the nucleon average potential (dot-dashed lines) and also the pion mean field (full lines).

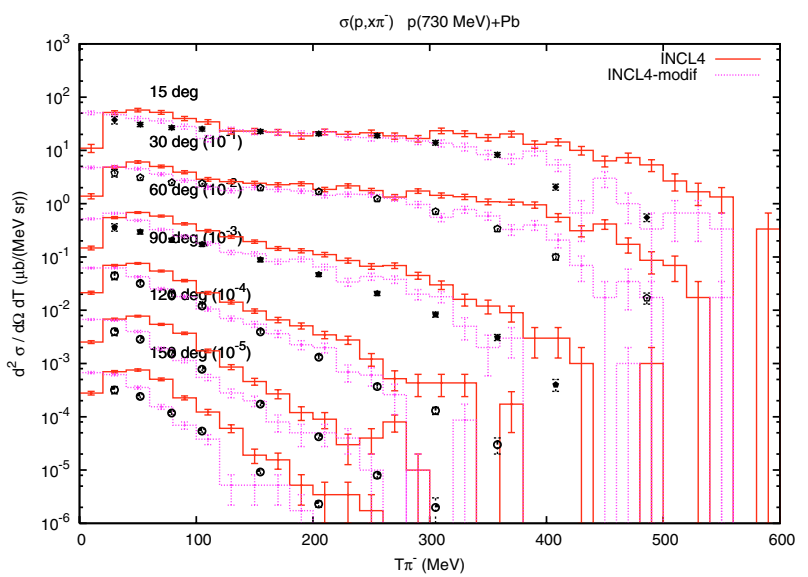

Fig. 3. Double differential negative pion production cross sections for $\mathrm{p}^{-208} \mathrm{~Pb}$ collisions at $730 \mathrm{MeV}$. Comparison between the predictions of the standard INCL4 model (red histograms), those of our modified model bearing on nucleon and pion average potentials (purple histograms) and the experimental data of ref. [10] (symbols).

dot-dashed lines in the lower panels of figure 2. This argument does not apply for the $\mathrm{Pb}$ target simply because, for heavy targets, pions may be produced in secondary collisions, which is not the case for such a light nucleus as ${ }^{12} \mathrm{C}$. The effect of adding the pion mean field can be understood along the same lines. In ${ }^{12} \mathrm{C}$, once a $\Delta$ is formed and decays, the emitted pion can escape easily. In an heavy nucleus, the pion can loose energy before leaving and may be trapped in the potential well.

We display in figure 3 a typical example of our results for double differential cross sections. One can see that the inclusion of all the modifications concerning the nucleon and the pion mean fields dramatically improves the theoretical description. The improvement in the high-energy tails of the pion spectra (even at lower energy for large angles) comes primarily from the energy-dependence of the nucleon average potential and the improvement at low energy (below $80 \mathrm{MeV}$ ) and small angles is due to the introduction of the pion mean field. This could be understood along the same lines as for the integrated cross sections.

\section{Conclusion}

We have studied the influence of some features of the mean field, both for nucleons and pions, on the theoretical description of spallation reactions. For the nucleons, we have considered the isospin-dependence and the energy-dependence of the nucleon mean field. These features are rather well documented by the phenomenology of the optical-model potential even though they are rarely included in simulation tools. The situation is less favorable for the pions, for which the opticalmodel phenomenology can hardly provide reliable indications on the pion average potential, at least in the nuclear interior. We have discussed the reasons for such a situation and even indicated that this quantity may not have a sound physical meaning. We therefore advocated to take this quantity as a pure phenomenological quantity, to be determined by fit to experimental data.

To evaluate the importance of these various features, we have implemented them on the standard version of the INCL4 model and compared the results with or without these features. Generally, the modifications are rather small. The most sensitive is the pion production cross section in p-induced reactions, whose description is considerably improved, curing a recurrent default of the INCL4 model.

This work has been partially done in the frame of the EU IP EUROTRANS project (European Union Contract No. FI6W-CT2004-516520). We acknowledge the EU financial support.

\section{References}

1. P. E. Hodgson, The Nucleon Optical Potential (World Scientific, Singapore, 1994).

2. A. Boudard, J. Cugnon, S. Leray, C. Volant, Phys. Rev. C 66, 044615 (2002).

3. C. Mahaux, R. Sartor, Adv. Nucl. Phys. 20, 1 (1991).

4. S. Nagamiya, M. Gyulassy, Adv. Nucl. Phys. 13, 201 (1984).

5. Th. Aoust, J. Cugnon, Phys. Rev. C 74, 064607 (2006).

6. S. Leray et al., Phys. Rev. C 65, 044621 (2002).

7. Th. Aoust, J. Cugnon, Eur. Phys. A 21, 79 (2004).

8. Th. Aoust et al. (these proceedings).

9. J.F. Crawford et al., Phys. Rev. C 22, 1184 (1980).

10. D.R.F. Cochran, Phys. Rev. D 3085 (1972). 